EPiC Series in Computing
Volume 69, 2020, Pages 206-213
Proceedings of 35th International Confer-
ence on Computers and Their Applications

\title{
SOC and SOH Monitoring Algorithms for Lithium Batteries Using Multilayer Neural Networks
}

\author{
Jong-Hyun Lee ${ }^{1}$, Hyun-Sil Kim ${ }^{2}$, and In-Soo Lee ${ }^{1 *}$ \\ ${ }^{1}$ Kyungpook National University School of Electronics Engineering, Daegu, Korea. \\ ${ }^{2}$ Naval Combat System PMO Agency For Defense Development, Jinhae, Korea. \\ whdugs8428@knu.ac.kr, shyla@add.re.kr, insooleedknu.ac.kr
}

\begin{abstract}
This paper presents a battery monitoring system using a multilayer neural network (MNN) for state of charge (SOC) estimation and state of health ( $\mathrm{SOH}$ ) diagnosis. In this system, the MNN utilizes experimental discharge voltage data from lithium battery operation to estimate $\mathrm{SOH}$ and uses present and previous voltages for SOC estimation. From experimental results, we know that the proposed battery monitoring system performs SOC estimation and $\mathrm{SOH}$ diagnosis well.
\end{abstract}

\section{Introduction}

Lithium batteries are used in various applications such as electric vehicles, portable devices, and energy storage systems (ESS) due to their high energy density, long life, and light weight (T. H. Cho et al., 2018). As the demand for lithium batteries increases, battery management systems (BMS) are being actively developed for efficient use of batteries. In order to implement BMS, it is necessary to monitor the battery's current state of charge (SOC) and state of health $(\mathrm{SOH})$. There are several methods for estimating SOC and SOH.

One SOC estimating method incorporates a current integral; another method employs an OCVSOC curve that indicates the relationship between the open-circuit voltage (OCV) and the SOC. The current integral accumulates measured current in real time. Although this method uses simple calculations and is intuitive, it requires knowing the battery's initial SOC and does not consider variables such as sensor error, battery age, and external temperature (K. W. Cheng et al., 2010). The OCV-SOC curve-based method requires OCV measurements. In general, it is impossible to measure the OCV in real time because it is necessary to make the measurement after the battery has rested for two hours or more while the circuit is open.

\footnotetext{
* Corresponding Author
} 
Battery $\mathrm{SOH}$ diagnosis methods can use a Kalman-filter approach. This method estimates the resistance of a battery's equivalent circuit (D. Andre et al., 2013) but is difficult to apply due to the complexity of its parameters and algorithms. Today, neural networks have been applied to problems such as pattern recognition, identification, and classification and are promising possibilities for BMS solutions.

In this paper, we propose a BMS that uses multi-layer neural networks (MNN) for SOC estimation and $\mathrm{SOH}$ diagnosis. In this BMS, the MNNs utilize discharge voltage data from prior battery operation as well as the present voltage as their inputs.

\section{Proposed SOC and $\mathrm{SOH}$ method}

The proposed SOC estimation and SOH diagnosis method are depicted in Figure 1. It consists of a system of two MNN's, one for SOC estimation and the other for SOH diagnosis. The MNN for SOC estimates the SOC from the current battery voltage and its previous voltage. On the other hand, the MNN for SOH receives the battery discharge voltage data for one cycle and diagnoses the battery status as normal, warning, or fault. The process of the proposed SOC estimation and SOH diagnostic method is shown in Figure 2.

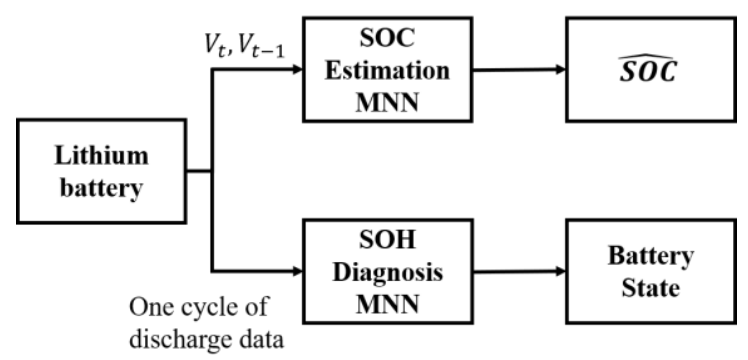

Figure 1: Block diagram of the proposed SOC estimation and $\mathrm{SOH}$ diagnosis method

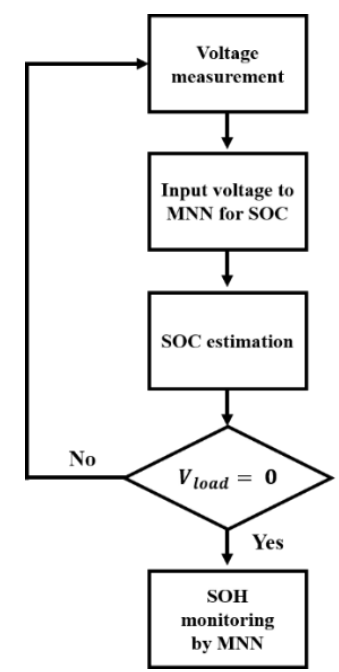

Figure 2: Process of the proposed SOC estimation and SOH diagnosis method 


\section{$2.1 \mathrm{SOC}$}

SOC is one of the most important parameters monitored by a BMS. There are several ways to determine a battery's SOC from its cell voltage, current, and temperature. The simplest method is to make a direct measurement of OCV or loaded voltage and then estimate the SOC from pre-stored discharge characteristics. However, this method influences the estimate due to small measurement errors (C. Zhang et al., 2018). SOC is defined in Equation (1):

$$
\text { SOC }=\frac{C_{\text {releasable }}}{C_{\max }} \times 100
$$

where $C_{\text {releasable }}$ is dischargeable charge capacity, $C_{\max }$ is maximum charge capacity, and SOC is the ratio of $C_{\text {releasable }}$ to $C_{\text {max }}$ expressed as a percentage.

\section{$2.2 \mathrm{SOH}$}

$\mathrm{SOH}$ is an indicator of decreased performance due to aging in lithium batteries, and the state of the battery is determined based on this. We define $\mathrm{SOH}$ as follows:

$$
S O H=\frac{C_{\text {actual }}}{C_{\text {fresh }}} \times 100
$$

where $C_{\text {actual }}$ is the capacity of the battery in its present state and $C_{\text {fresh }}$ is the battery's rated capacity as stated on the manufacturer's datasheet. A battery is in a fault state when its SOH reaches $80 \%$ (Y. Xing et al., 2011). In this paper, we defined $100 \% \sim 90 \%$ capacity of the rated capacity as normal, $90 \sim 80 \%$ of the rated capacity as a warning, and less than $80 \%$ of the capacity as a fault.

\section{$2.3 \mathrm{SOH}$}

An MNN is the most basic learning model in multi-layer learning and refers to a model with two or more hidden layers. It allows a high level of abstraction by combining various nonlinear transform techniques with more hidden layers than found in existing neural networks. The MNN used in this paper consists of one input layer, two hidden layers, and one output layer as shown in Figure 3.

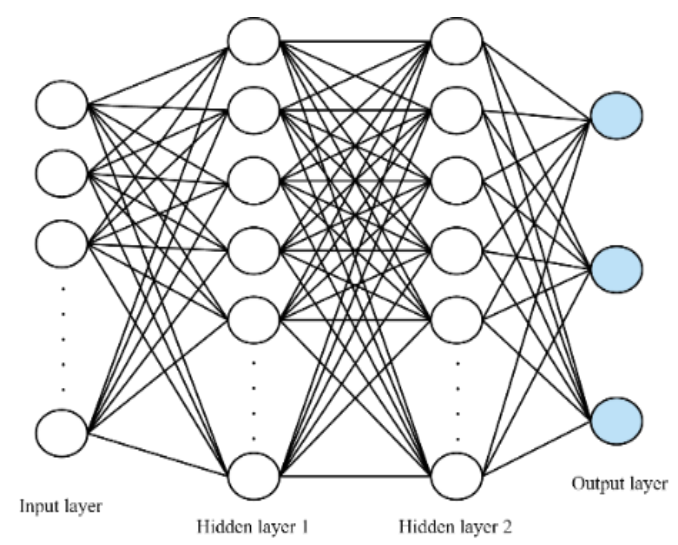

Figure 3: Structure of a Multilayer Neural Network 
The learning optimization method was stochastic gradient descent (SGD) for the SOC estimation MNN; it was RMSprop for the SOH diagnosis MNN. SGD and RMSprop are governed by Equations (3) to (5):

$$
\theta_{t+1}=\theta_{t}-\eta \frac{\partial}{\partial \theta_{t}} \nabla_{\theta} f_{t}\left(\widehat{T}_{t}, \theta_{t}\right), \quad \widehat{T}_{t} \subset T
$$

where $\theta$ is a parameter of the network, $\eta$ is the learning rate $=0.01, f(\theta)$ is the cost function, $\nabla_{\theta} f(\theta)$ is the gradient of the network, $\mathrm{t}$ is a time step, $\widehat{T}_{t}$ is the batch size of the training data, and $T$ is the training data set.

$$
\begin{gathered}
G_{t}=\gamma G+(1-\gamma)\left(\nabla_{\theta} f_{t}\left(\theta_{t}\right)\right)^{2} \\
\theta_{t}=\theta_{t-1}-\sqrt{\frac{\eta}{G_{t}-\epsilon}} \cdot \nabla_{\theta} f_{t}\left(\theta_{t-1}\right)
\end{gathered}
$$

where $\gamma$ is the forgetting factor, typically 0.9 . If the network parameter is $\mathrm{k}$, then $\mathrm{G}_{t}$ is a $\mathrm{k}$-dimensional vector that stores the sum of squares of the gradient to which each variable moves until time step t. $\eta$ is the learning rate and is set to $0.001 . \epsilon$ is a small value of about $10^{-4}$ to $10^{-8}$ to prevent division by 0 . The number of hidden layers, the learning rate and the forgetting factor value for $\mathrm{SOC}$ and $\mathrm{SOH}$ were selected through a trial-and-error process.

The activation function for the MNN nodes was the rectified linear unit (ReLU) function as defined in Equation (6):

$$
f(x)=\left\{\begin{array}{l}
x, \text { for } x>0 \\
0, \text { for } x \leq 0
\end{array}\right.
$$

\section{Experiment and Results}

The experimental setup is shown in Figure 4. Figure 4-(1) is an electronic load that is replaced with a power supply when charging the battery. Figure 4-(2) is the battery system consisting of the battery, current and voltage sensors, and a microcontroller to communicate with the PC. Figure 4-(3) is the PC that receives battery voltage and current data to monitor SOC and SOH.

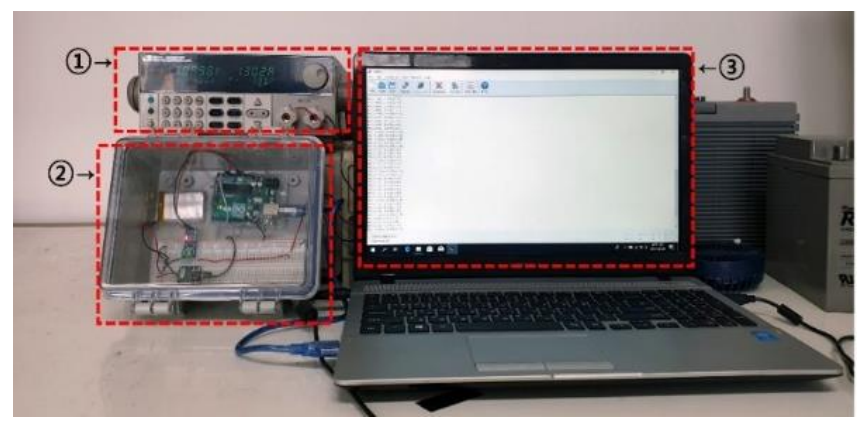

Figure 4: Experimental configuration

The 2-128-64-1 MNN (2 input nodes, 128 hidden nodes in hidden layer 1, 64 hidden nodes in hidden layer 2, and 1 output node) structure used for SOC estimation shown in Figure 5. The network was trained with SGD optimization, and each layer's nodes were activated with the ReLU function. 


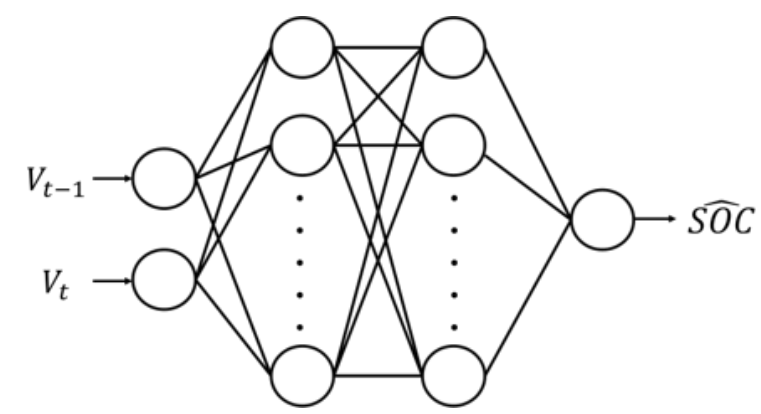

Figure 5: The structure of the MNN for SOC estimation

The 3600-256-256-3 MNN structure for SOH diagnosis is shown in Figure 6. The output is 1 when the battery is in a normal state, 2 when the battery is in a warning state, and 3 when the battery is in a fault state. RMSprop was used for optimized training. The ReLU function activated each hidden layer.

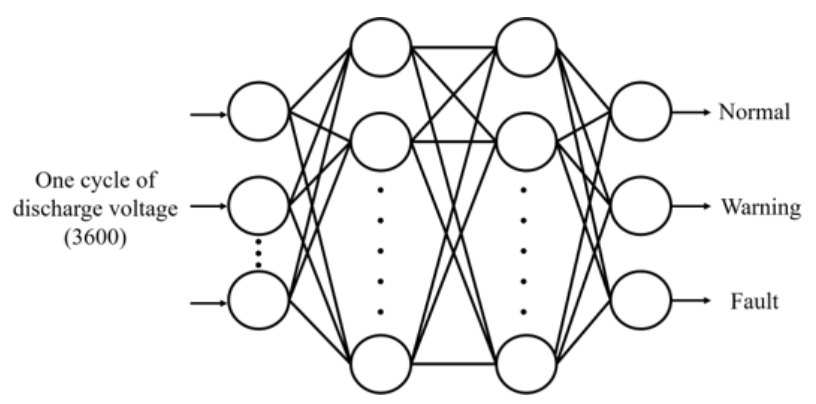

Figure 6: The structure of the MNN for SOH diagnosis

In order to evaluate the performance of the proposed system, the discharge voltage data of the battery operated as an experiment was used. The training data are plotted in Figure 7 (a). The test used the data in Figure 7 (b).

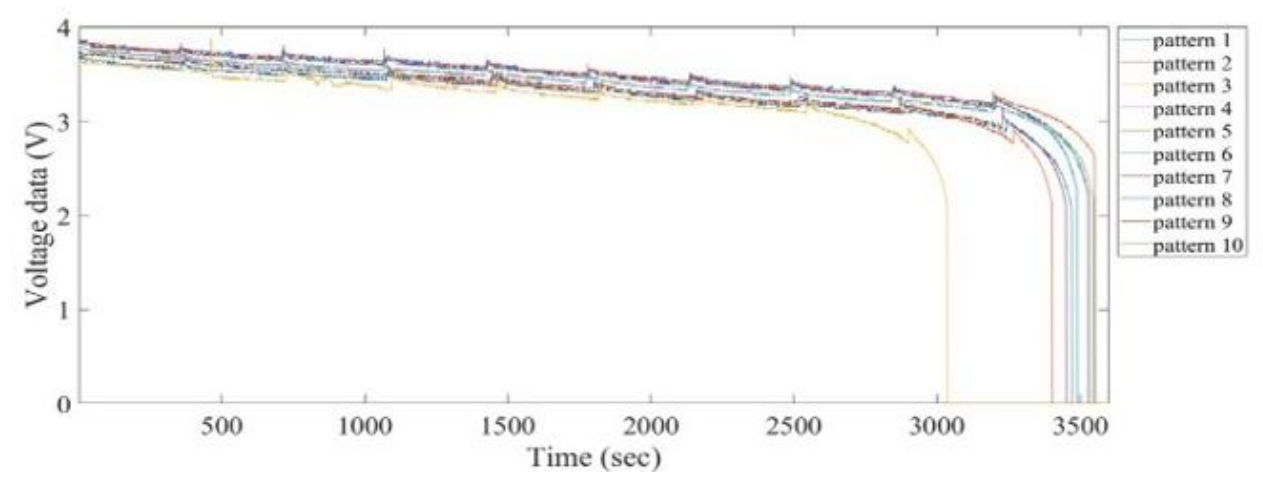

(a) Battery discharge voltage data used in the test 


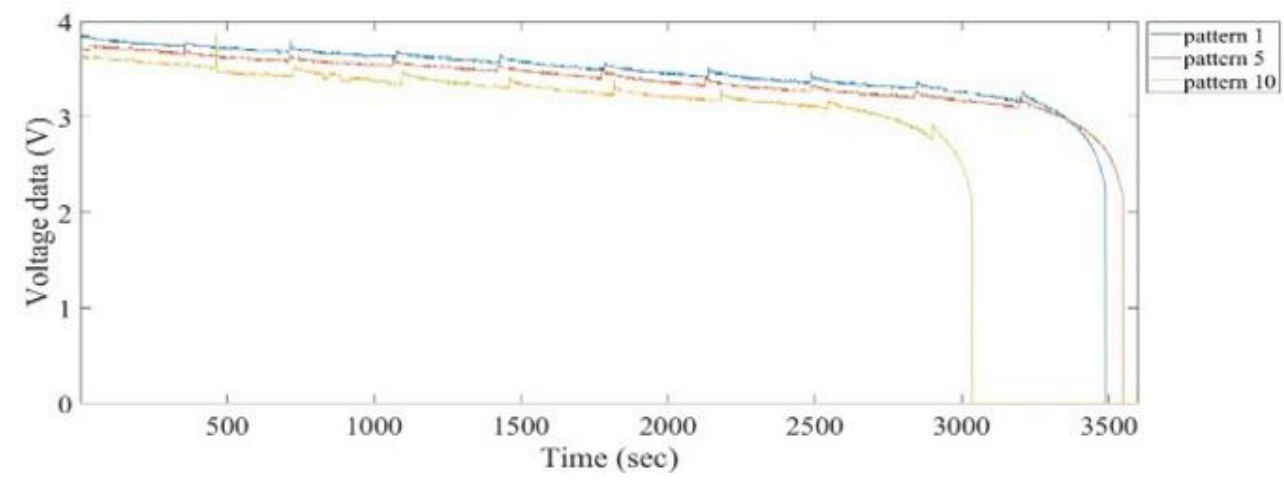

(b) Battery discharge voltage data used in the test

Figure 7: Lithium battery discharge graph for MNN

The SOC estimation results are shown in Figure 8. The estimates used the previous and current voltages as inputs to the SOC MNN. Pattern 1 and pattern 5 showed errors of $2 \%$, respectively, but pattern 10 failed to estimate SOC to any usable degree. This is because pattern 10 is the discharge characteristic of an aged battery.

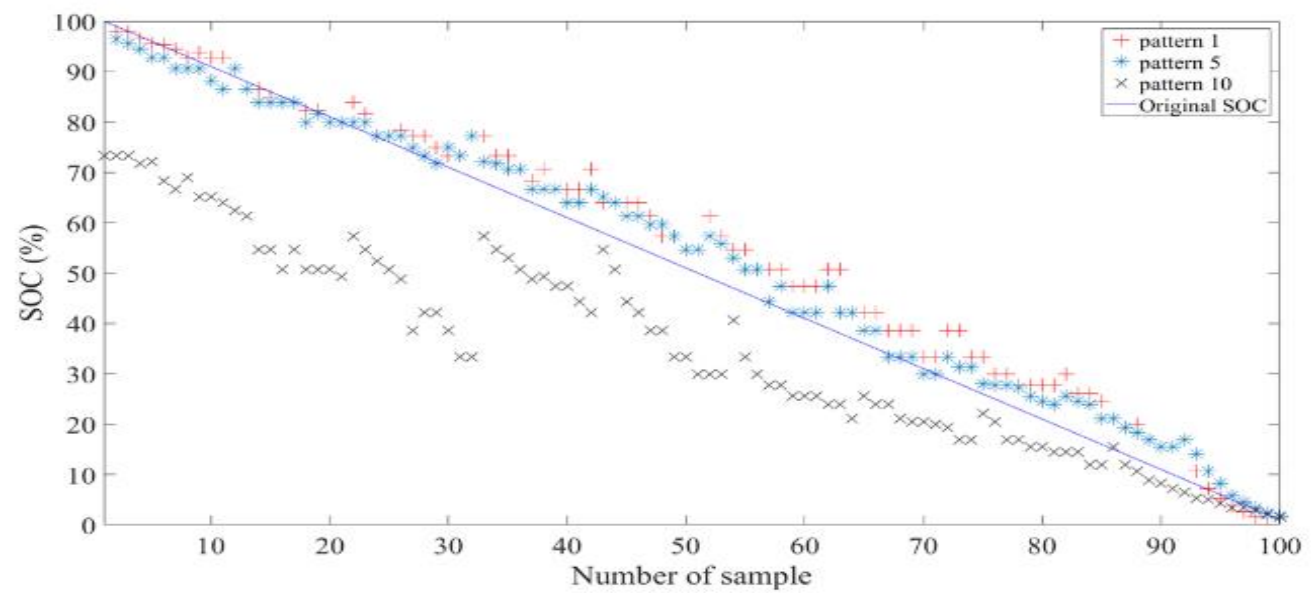

Figure 8: SOC estimation result

The following is the SOH diagnosis result. The patterns 1 and 5 results are normal and pattern 10 produced a warning. The SOH diagnosis used one cycle of discharge data as inputs to the MNN. These results are shown in Figure 9. This shows that the proposed system accurately diagnoses the SOH of a battery. 


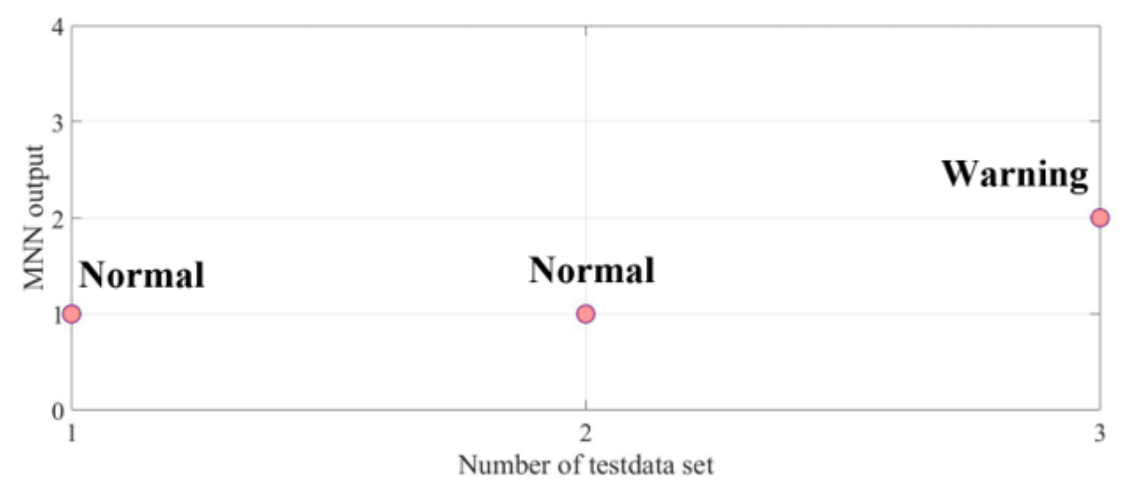

Figure 9: $\mathrm{SOH}$ diagnosis result

\section{Conclusion}

In this study, we propose a lithium battery module SOC and $\mathrm{SOH}$ monitoring system using MNNs. The proposed system uses data from a battery operated in a general environment. The results show that the MNNs correctly diagnose the battery state. The SOC of the initial battery was well estimated within an error range of $2 \%$, but the SOC of an aged battery was not well estimated. The next study will research methods for estimating the state of an aging battery.

Future work includes comparing neural network approach with support vector machines (SVM) or adaptive neural fuzzy inference (ANFIS) techniques as well as applying the proposed method to real systems.

\section{Acknowledgments}

This research was supported by the Basic Science Research Program through the National Research Foundation of Korea (NRF) funded by the Ministry of Education (No. 2019R1I1A3A01058319).

This study was also supported by the BK21 Plus project funded by the Ministry of Education, Korea (No. 21A20131600011).

\section{References}

T. H. Cho, H. R. Hwang, J. H. Lee and I. S. Lee. (2018). Comparison of Intelligent Methods of SOC Estimation for Battery of Photovoltaic System. International Journal of Advanced Computer Science and Applications 9(9) (pp. 49-56).

K. W. Cheng, E, B. P. Divakar, H. Wu, K. Ding, and H. F. Ho, (2010). Battery-management system (BMS) and SOC development for electrical vehicles. IEEE transactions on vehicular technology 60(1) (pp. 76-88)

D. Andre, C. Appel, T. Soczka-Guth and D. U. Sauer. (2013). Advanced mathematical methods of SOC and SOH estimation for lithium-ion batteries, Journal of Power Sources 224 (pp. 20-27). 
C. Zhang, W. Allafi, Q. Dinh, P. Ascencio, and J. Marco. (2018). Online estimation of battery equivalent circuit model parameters and state of charge using decoupled least squares technique. Energy 142 (pp. 678-688).

Y. Xing, E. W. Ma, K. L. Tsui, and M. Pecht. (2011). Battery management systems in electric and hybrid vehicles. Energies 4(11) (pp. 1840-1857). 